\title{
Retarding viscous Rayleigh-Taylor mixing by an optimized additional mode
}

\author{
C.Y. Xie, J.J. Tao, ${ }^{*}$ and Z.L. Sun \\ CAPT-HEDPS, IFSA Collaborative Innovation Center of MoE, \\ SKLTCS, Department of Mechanics and Engineering science, \\ College of Engineering, Peking University, Beijing, 100871, China \\ J. $\mathrm{Li}$ \\ Department of Engineering, University of Cambridge, Cambridge CB2 1PZ, UK
}

(Dated: December 19, 2016)

\begin{abstract}
The Rayleigh-Taylor (RT) mixing induced by random interface disturbances between two incompressible viscous fluids is simulated numerically. The ensemble averaged spike velocity is found to be remarkably retarded when the random interface disturbances are superimposed with an optimized additional mode. The mode's wavenumber is selected to be large enough to avoid enhancing the dominance of long-wavelength modes, but not so large that its saturated spike and bubble velocities are too small to stimulate a growing effective density-gradient layer suppressing the long-wavelength modes. Such an optimized suppressing mode is expected to be found in the RT mixing including other diffusion processes, e.g. concentration diffusion and thermal diffusion.
\end{abstract}

PACS number(s): 47.20.Ma, 47.20.Ky

\section{INTRODUCTION}

The Rayleigh-Taylor instability (RTI) occurs when a heavy fluid is laid on a light fluid in a gravitational field or the interface is accelerated to the heavier one $[1,2]$. RTI is important in a wide variety of application$\mathrm{s}$ including inertial confinement fusion[3], astrophysical phenomena $[4,5]$, ultrathin foil accelerated by the radiation pressure[6], and gas-particle mixtures[7]. It has been shown that the growth of an unstable RT mode may be retarded by surface tension and viscosity[8], concentration diffusion[9], rotation[10] or the bi-mode interaction[11]. When RTI arises from random interface perturbations (initial disturbances with banded spectra), the highly disordered mixing zone is dominated by large scale structures, and its width increases as $h_{b} \sim \alpha_{b} A g t^{2}$ [12-18], where $A$ is the Atwood number, $g$ is gravity, $h_{b}$ and $\alpha_{b}$ are the penetration depth and the growth rate parameter of bubbles, respectively. Since no fluid is completely inviscid, the momentum-diffusion (viscous) effect has been an important topic for theoretical [19, 20], experimental [21] and numerical [22] studies of RTI and RT mixing. It has been revealed numerically and experimentally that addition of a single or multi-modes with long wavelength(s) can enhance the RT mixing, i.e. increasing the instability penetration [14] and the growth rate parameter $\alpha_{b}[18,23]$. However, it is still unknown by far whether there exists an additional mode that is optimized to suppress utmostly the viscous RT mixing induced by random initial disturbances, and this is the main motivation of this paper.

*jjtao@pku.edu.cn
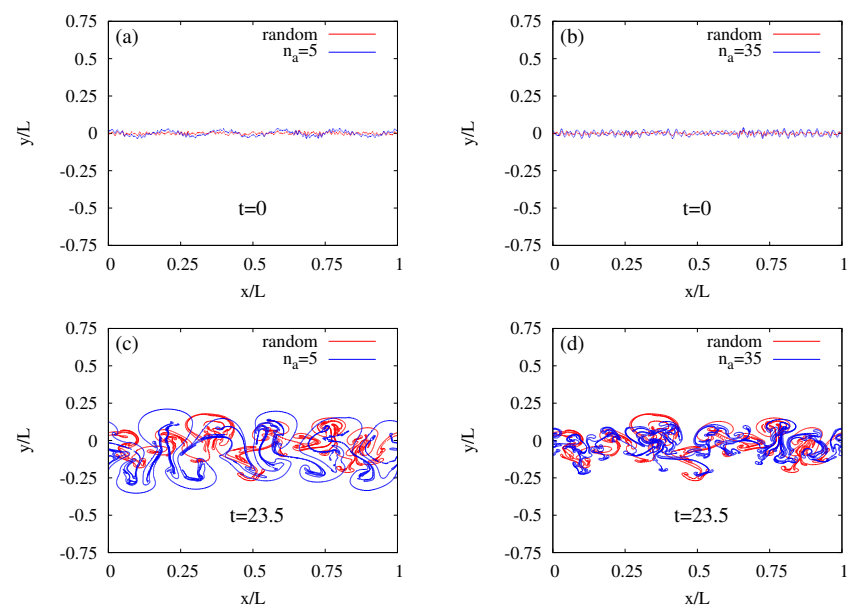

FIG. 1. Temporal evolutions of the fluid interfaces disturbed initially by random perturbations with (blue) and without (red) a superimposed additional mode of wavenumber $n_{a}$.

\section{OPTIMIZED SUPPRESSING MODE}

The two-dimensional incompressible viscous NavierStokes equations are solved by a projection method in the Cartesian coordinates with $x^{*}$-axis lying on the undisturbed interface between the upper heavier fluid of density $\rho_{2}$ and the lower lighter fluid of density $\rho_{1}$. The gravity $g$ points to the contradict direction of $y^{*}$, and the characteristic length and time scales are $\left(\nu^{2} / g\right)^{1 / 3}$ and $\left(\nu / g^{2}\right)^{1 / 3}$ respectively, where $\nu=\left(\mu_{1}+\mu_{2}\right) /\left(\rho_{1}+\rho_{2}\right), \mu_{1}$ and $\mu_{2}$ are the dynamic viscosities of the lower and the upper fluids, respectively.

The interface is tracked with the VOF method [24] and the pressure Poisson equation is solved by the multi-grid method. The ratio between the width $L$ to the hight $H$ of the computational domain is 1:4. A uniform mesh 
$2048 \times 8192$ is tested by comparing results with those of $1024 \times 4098$ without significant difference. In addition, good consistency is obtained between the growth rate predicted by the linear stability theory of viscous RTI [25] and the simulation value based on the mesh $2048 \times 8192$. Therefore, $2048 \times 8192$ is used in the simulations. The widths of the mixing zone studied in this paper are less than $2 L$, so no-slip boundary conditions are applied on the top and the bottom and periodic conditions are used in the horizontal direction. For simplicity, it is assumed in the following simulations that both fluids have the same kinematic viscosity $\mu_{1} / \rho_{1}=\mu_{2} / \rho_{2}$, and the Atwood number $A=\left(\rho_{2}-\rho_{1}\right) /\left(\rho_{1}+\rho_{2}\right)=0.65$.

The fluids are initially quiescent with a random interface displacement in the vertical direction

$$
h_{\text {ran }, 0}=\delta \sum_{n=1}^{100} \cos \left(\frac{2 n \pi}{L} x+\phi_{n}\right) \text {, }
$$

where $\phi_{n}$ is a random phase lag in $[0,2 \pi]$ [26]. The additional single mode is $a \cos \left(2 n_{a} \pi x / L\right)$ and $n_{a}$ is an integer wavenumber. In order to examine whether the addition mode could suppress the RT mixing, $\delta$ and $a$ are assumed to be constants of $0.001 L$ and $0.02 L$, respectively. Accordingly, the amplitude of $h_{\text {ran }, 0}$ is about $0.02 L$ for different random cases.

Along with the growth of perturbations, the lighter fluid bubbles up while the heavier one penetrates into the lighter fluid forming spikes. It is shown in Fig.1 that the width of the RT mixing zone increases when the initial random perturbations are superposed with a low wavenumber mode of $n_{a}=5$ [Fig.1(a), (c)], but decreases for an additional mode with a large wavenumber $n_{a}=35$ as shown in Fig.1(b) and (d). The maximum heights (above/below the initial unperturbed interface) of bubbles and spikes are referred as the bubble and the spike penetration depths $h_{b}$ and $h_{s}$, respectively. Considering that the initial amplitude of the superposed case is about twice larger than that of the random perturbations, the suppression effect of large wavenumber mode on both $h_{b}$ and $h_{s}$ is substantial.

In order to look for the most effective wavenumber to suppress the RT mixing, 50 independent randomly perturbed interfaces and different additional modes are tested, and the ensemble-averaged results are shown in Fig. 2. In comparison with the random cases, different wavenumbers $n_{a}$ of the additional modes lead to different evolution scenarios of $h_{s}$ as shown in Fig.2(a). The spike velocity $v_{s}=d h_{s} / d t$ for the case without additional mode can be fitted with a straight dashed line in Fig.2(b), indicating the self-similar stage where dimensionless parameters satisfy $v_{s} \sim A t$. At the initial stage of RT mixing, smaller $n_{a}$ produces larger $v_{s}$ and hence wider mixing zone than those of the random case, while increasing $n_{a}$ decreases $v_{s}$ and then suppresses the mixing. However, when $n_{a}$ is very large, it is shown in Fig.2(b) that the spike velocity for the superposed case with $n_{a}=60$ becomes higher than that of $n_{a}=35$ as $t>18$, indicating that there
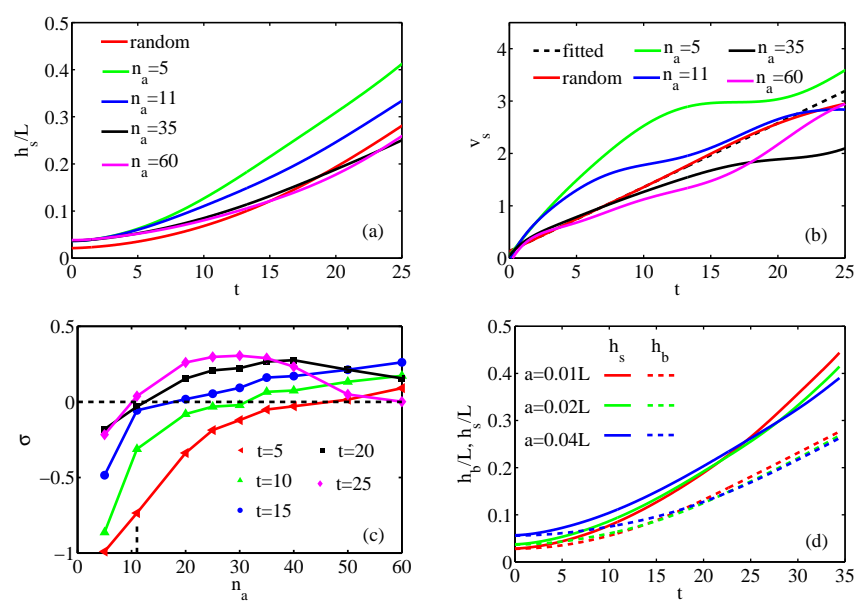

FIG. 2. Temporal evolutions of the ensemble-averaged values of the spike penetration $h_{s}$ (a) and the spike velocity (b) with and without the superposed additional modes. (c) The velocity suppression factor $\sigma$ as a function of the additional wavenumber $n_{a}$ at different time. The dashed vertical line in (c) represents the wavenumber of the most unstable linear viscous mode. (d) Temporal evolution of $h_{s}$ and $h_{b}$ for $n_{a}=35$ at different initial amplitude $a / L=0.01,0.02,0.04$, respectively.

must exist the most effective suppressing mode with $n_{a}$ less then 60. Later on, the spike penetration depth $h_{s}$ of $n_{a}=60$ is also larger than that of $n_{a}=35$ at $t=25$ (Fig.2a), but is still smaller than the $h_{s}$ of the random initial perturbation case.

The suppression effects of different additional modes are evaluated with the velocity suppression factor $\sigma=$ $1-v_{s, a} / v_{s, r}$, where $v_{s, a}$ and $v_{s, r}$ are the ensembleaveraged spike velocities with and without the additional modes, respectively. Consequently, $\sigma>0$ and $\sigma<0$ represent a suppression and an enhancing effects, respectively. As shown in Fig.2(c), $\sigma<0$ for small $n_{a}$, especially as $n_{a}<11$, the wavenumber of the most unstable linear mode. This phenomenon is consistent with the previous observation [14]. The marginal $n_{a}$ above which $\sigma>0$ decreases quickly from 50 at $t=5$ to 11 at $t=20$, and at $t=25$ a maximum $\sigma$ of $31 \%$ is reached at $n_{a}=30$, the wavenumber optimized for the most suppressing effect at this instant. It is also shown in Fig.2(d) that the additional mode with larger amplitude has a stronger retardation effect on the spike and bubble penetrations at the late mixing stage than that with smaller amplitude.

\section{MECHANISM OF THE OPTIMIZED SUPPRESSION}

The optimized suppression mechanism includes two aspects. First, the dominance of long-wavelength or lowwavenumber modes is enhanced by the viscous diffusion. For inviscid fluids, the linear growth rate of an initial perturbation as $h_{0} \cos (2 \pi n x / L)$ is proportional to $\sqrt{n}$, so a 

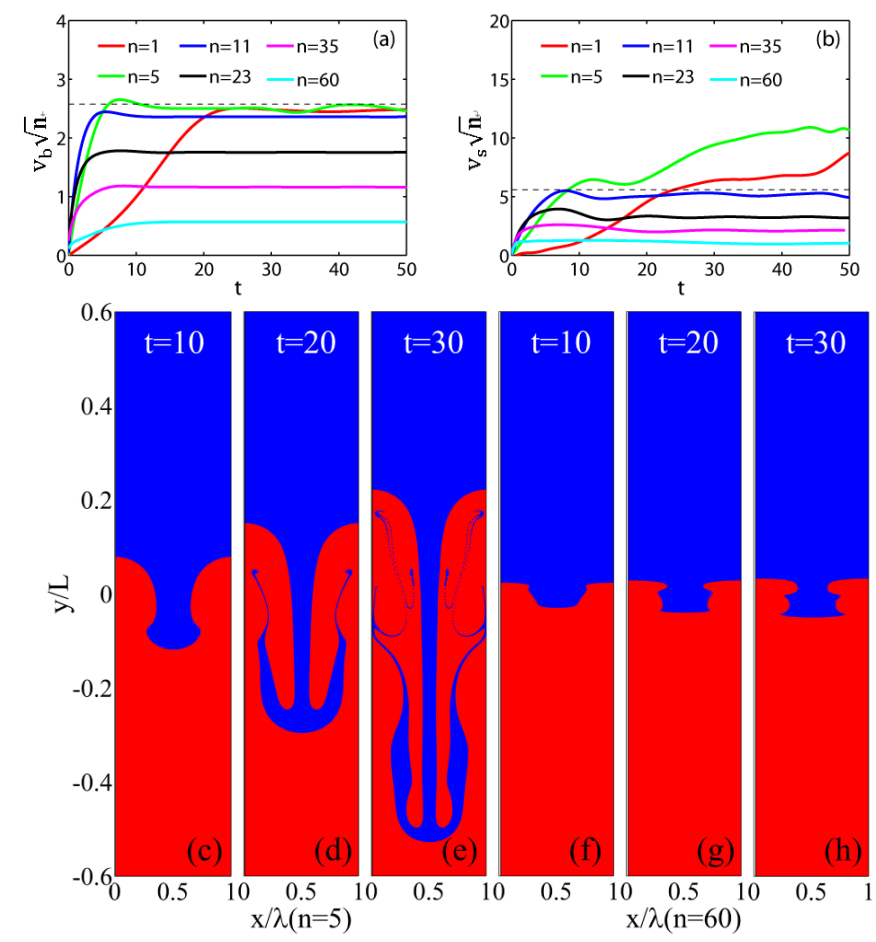

FIG. 3. Wavenumber dependence of the interface evolution for a single viscous RT mode. The bubble velocity and the spike velocity multiplied with $\sqrt{n}$ are compared with their inviscid saturated values $v_{b} \sqrt{n}=\sqrt{\frac{A L}{3 \pi(1+A)}}=2.576$ and $v_{s} \sqrt{n}=\sqrt{\frac{A L}{3 \pi(1-A)}}=5.593[27]$ shown by the dashed lines in (a) and (b), respectively. The temporal evolution of a viscous mode interface with a large wavenumber $n=60$ is shown in (f)-(h), and the results for low wavenumber mode with $n=5$ are shown in (c)-(e) as references.

mode with large $n$ grows fast and reaches its saturated velocity [27] soon, while the mode with small $n$ still grows exponentially and then dominates the late stage [28]. The dispersion relation for viscous RTI [25] was simplified [8] to obtain an approximate expression of the dimensionless growth rate as $\sqrt{k A+k^{4}}-k^{2}$, where $k=2 n \pi / L$. With the increase of $n$, the viscous linear growth rate increases first similar as the invscid case, then decreases after reaching its maximum value at $n=11$ for $A=0.65$. Therefore, at the initial stage modes with wavenumbers larger than 11 have been suppressed by viscous diffusion, and the dominance of long-wavelength mode $(n<11)$ during a viscous RT mixing becomes more remarkable in comparison with the inviscid case. As shown in Fig. 1(c), the instantaneous interface fluctuation at the late stage of mixing is dominated by large-scale structures with a wavenumber around 5 , the value of the additional mode. The ensemble averaged spike velocity with a largewavelength additional mode $\left(n_{a}=5\right)$ increases with a higher grow rate parameter than that of the case without the additional mode (Fig.2b), a phenomenon consistent with previous $2 \mathrm{D}$ and $3 \mathrm{D}$ simulations $[14,18]$.
Second, the suppression of an additional mode with a very high wavenumber on the low-wavenumber modes is weakened by the viscous diffusion. It is proposed for inviscid fluids [11] that the mushroom shape at the spike tips of short-wavelength mode imposes effectively a density gradient layer on the effective interface of the long-wavelength mode with wavenumber of $k$ and modifies the linear growth rate of long-wavelength mode as $\gamma_{e f f}=\sqrt{A k /(1+k l)}$. The density gradient scale length $l$ is evaluated as $l \sim w a_{s} k_{s}$, where $w$ is the mushroom width and $a_{s}$ and $k_{s}$ are the amplitude and the wavenumber of the short-wavelength mode, respectively. This approximation requires $k_{s}>>k$ and the underlying mechanism is applicable for viscous RT mixing as well, i.e. larger $l$ leads to smaller $\gamma_{e f f}$ or larger suppression effect. Assuming $w \sim 1 / k_{s}$ then we get $l \sim a_{s}$. At the initial stage the amplitudes of all modes are small, an additional mode with a higher wavenumber can suppress more modes with low and moderate wavenumbers, and hence higher $n_{a}$ leads to more effective suppression just as shown by the data at $t=5$ in Fig.2(c). At the late stage, nonlinear evolution of bubbles leads to saturated velocities, which are very close to its inviscid limit[27] for low wavenumber modes as shown in Fig.3(a). Since in this paper the Atwood number is relatively large $A=0.65$, only the spike velocity shows the reacceleration phenomenon (Fig. $3 \mathrm{~b}$ ), which is consistent with the previous simulations of single mode [29]. For high-wavenumber modes $(n>11)$, the reacceleration of spikes disappear because the viscous diffusion suppresses the formation of Kelvin-Helmholtz structures on the sides of spikes [Fig.3(f)-(h)]. More importantly, the saturated velocities of both bubbles and spikes decrease remarkably with the increase of wavenumber as $n>11$ (Fig.3a,b), leading to a smaller amplitude $a_{s}$ in comparison with the inviscid case. Therefore, if the wavenumber of the additional mode is too large, viscous diffusion will dramatically retards the growth of $a_{s}$ and $l$, enlarging $\gamma_{e f f}$ and weakening its suppression effect on long-wavenumber modes. As a result, at any instant during the nonlinear mixing process, there must be an additional mode with the optimized wavenumber to utmostly suppress the dominant long-wavelength mode.

The RT mixing process induced by random interface disturbances is characterized with multiple time and length scales. By introducing the fluid mixture fraction $\rho^{\prime}=\left(\rho-\rho_{1}\right) /\left(\rho_{2}-\rho_{1}\right)$, we can calculate the ensembleaveraged density spectrum $\bar{E}_{\rho}$ at the original undisturbed interface as shown in Fig.4, and for each sample case we have $\int E_{\rho}(n) d n=\overline{\rho^{\prime 2}}$. Without the additional mode, the dominant wavenumber (the wavenumber of the spectrum peak) for random initial disturbances is around the value of the most unstable linear mode $(n=11)$ at the early stage (Fig.4a), and then becomes smaller and smaller with time because more and more high-wavenumber modes reach their saturated velocities. In the meantime, the flow around the original interface becomes fullydeveloped turbulence and the inertial subrange labelled 

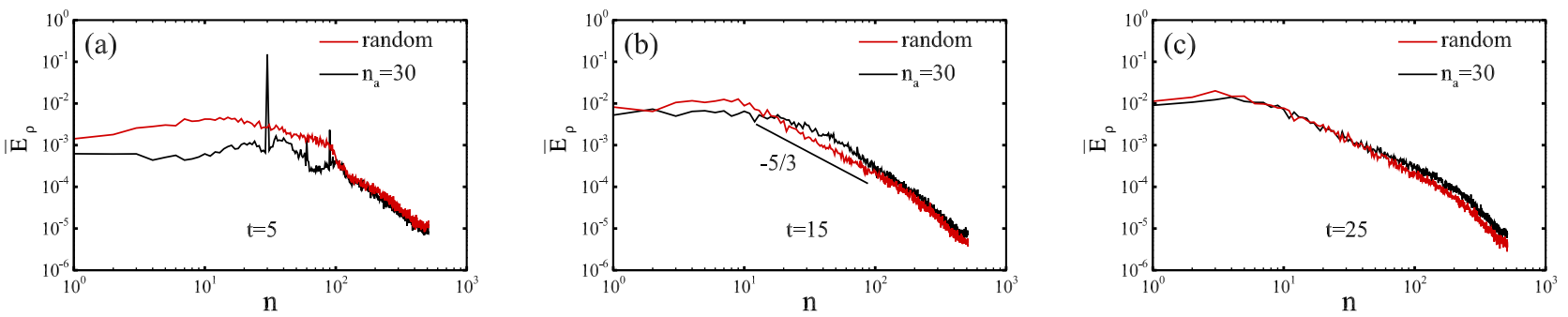

FIG. 4. The ensemble-averaged density spectra calculated at the mid-plane (the original interface) and different times for cases with (black) and without (red) the additional mode.

by the $-5 / 3$ law [30] emerges in the spectrum (Fig.4b). When the additional mode (e.g. $n_{a}=30$ ) is added initially, its higher harmonics are produced almost simultaneously (Fig.4a). Though the additional mode and its harmonics lost their dominance in the following stage (e.g. Fig. $4 \mathrm{~b}$ ) and the spectrum curve becomes smooth, their influence is continued. It is shown in Fig.4(b) and (c) that in the later stage the modes with large wavenumbers are stimulated by the additional mode and its harmonics, and the spectrum curves at the large-wavenumber range are higher than their counterparts without the additional mode, while the spectrum peaks at the low-wavenumber range are suppressed and hence the growth of the mixing zone is retarded.

\section{CONCLUSIONS}

By numerical simulations and statistical analysis, it is shown that the growth of RT mixing zone can be substantially retarded by superimposing an optimized additional mode on its random initial perturbations. The additional mode and its harmonics enhance large wavenum- ber modes to form an effective density-gradient layer for the dominant long wavelength modes and hence decrease their growth rates. Since viscous diffusion undermines the effectiveness of the additional mode with too large wavenumber, optimized wavenumber can be found by looking for the maximum velocity-suppression factor. Though different Atwood numbers, viscosity ratios, initial disturbance amplitudes and three-dimensionality influence the selection of the additional mode and worth further investigations, the optimized suppression mechanism caused by the viscous diffusion is universal, and is expected to be applied in the RT mixing processes accompanied with thermal diffusion and concentration diffusion.

\section{ACKNOWLEDGMENTS}

The authors thank X.T. He and W.H. Ye for the inspiring discussions on Rayleigh-Taylor instability. This work has been supported by National Natural Science Foundation of China (No.11225209, 11490553 and 11521091).
[1] L. Rayleigh, Proc. London Math. Soc. 14, 170 (1883).

[2] G. I. Taylor, Proc. R. Soc. London, Ser. A 201, 192 (1950).

[3] S. P. Regan et al., Phys. Plasmas 19, 056307 (2012).

[4] C. C. Joggerst, A. Almgren, and S. E. Woosley, Astrophys. J. 723, 353 (2010).

[5] G. S. Novak, J. P. Ostriker, and L. Ciotti, Astrophys. J. 737, 26 (2011).

[6] C. A. J. Palmer et al., Phys. Rev. Lett. 108, 225002 (2012).

[7] K. Balakrishnan, Phys. Fluids 26, 043303 (2014).

[8] R. Bellman and R.H. Pennington, Q. Appl. Math. 12,151-162, (1954).

[9] R.E. Duff, F.H. Harlow, and C.W. Hirt, Phys. Fluids 5,417-425 (1962).

[10] J.J. Tao, X.T. He, W.H. Ye, and F.H. Busse, Phys. Rev. E 87, 013001 (2013).

[11] D. Ofer et al., Phys. Plasmas 4, 3549 (1992).
[12] C. Cherfils, and K. O. Mikaelian, Phys. Fluids 8, 522 (1996).

[13] G. Birkhoff, University of California Report No. LA1862, (1955).

[14] D. L. Youngs, Physica D 12, 32 (1984).

[15] K. I. Read, Physica D 12, 45 (1984).

[16] N. Freed, D. Ofer, D. Shvarts, and S.A. Orszag, Phys. Fluids A 3,912 (1991).

[17] M.B. Schneider, G. Dimonte, and B. Remington, Phys. Rev. Lett. 80,3507 (1998).

[18] G. Dimonte et al., Phys. Fluids 16, 1668 (2004).

[19] S. Chandrasekhar, Hydrodynamic and Hydromagnetic Stability, Oxford University Press, London (1961).

[20] K.O. Mikaelian, Phys. Rev. E 93, 023104 (2016).

[21] M.S. Roberts, and J.W. Jacobs, J. Fluid Mech. 787, 5083 (2016).

[22] M.A. Gallis, T.P. Koehler, J.R. Torczynski, and S.J. Plimpton, Phys. Rev. Fluids 1, 043403 (2016). 
[23] A. Banerjee and M. J. Andrews, Int. J. Heat Mass Transf. 52, 3906 (2009).

[24] D. Gueyffier, J. Li, A. Nadim, R. Scardovelli, and S. Zaleski, J. Comput. Phys. 152, 423 (1999).

[25] W.J. Harrison, Proc. London Math. Soc. 6, 396-405 (1908).
[26] T. T. Clark, Phys. Fluids 15, 2413 (2003).

[27] V. N. Goncharov, Phys. Rev. Lett. 88, 134502 (2002).

[28] D. Ofer et al., Phys. Plasmas 3, 3073 (1996).

[29] P. Ramaprabhu et al., Phys. Fluids 24, 074107 (2012).

[30] W.J.T. Bos, B. Kadoch, K. Schneider, and J.-P. Bertoglio Phys. Fluids 21, 115105 (2009). 